\title{
Caractérisation biochimique de l'algue verte Scenedesmus abundans : influence des conditions de culture
}

\author{
M. Tahiri ${ }^{1}$, \\ A. Benider ${ }^{1}$, \\ M. Belkoura ${ }^{1,2}$
}

A. Dauta ${ }^{3}$

Mots-clés : Scenedesmus abundans, protéines, glucides, lipides, température, lumière, azote, phase de croissance, taux de croissance.

Dans le but d'évaluer les perspectives de valorisation de l'algue verte Scenedesmus abundans Chlorophyceae, sa composition biochimique a été réalisée sur des cultures soumises à différentes conditions environnementales. Parmi les facteurs retenus, la température a le plus d'influence. Les teneurs en protéines sont maximales à $15^{\circ} \mathrm{C}$, celles des composés lipidiques et glucidiques à 25 et $35^{\circ} \mathrm{C}$ respectivement. L'intensité lumineuse n'affecte pas la composition biochimique globale, si ce n'est une baisse d'environ $20 \%$ des substances lipidiques, sous un éclairement de $300 \mu \mathrm{mol}$ quanta. $\mathrm{m}^{-2} . \mathrm{s}^{-1}$. De même, la source azotée a peu d'effet significatif sur les teneurs des 3 constituants cellulaires. L'augmentation de la concentration en azote induit un accroissement des teneurs en protéines et en lipides avec cependant une réduction de la production de biomasse, au-delà de 400 mg.1.$^{-1}$ de $\mathrm{KNO}_{3}$. Des modifications de la composition biochimique de Scenedesmus abundans en fonction du stade de croissance ont également été observées. En phase de croissance stationnaire, ces modifications se résument en une réduction marquée de la teneur en protéines et, dans une moindre mesure, de la teneur en lipides, accompagnée d'une accumulation importante des hydrates de carbone. Les résultats sont analysés en tenant compte de la notion de rendement de production de chacun des trois types de composé.

\section{Biochemical composition of the green algae Scenedesmus abundans : effects of culture conditions}

Keywords : Scenedesmus abundans, proteins, carbohydrates, lipids, temperature, light, nitrogen, growth phase, growth rate.

To evaluate aspects of enhancement of the green algae Scenedesmus abundans (Chlorophyceae), its biochemical composition was examined in cultures exposed to different environmental conditions. Amongst the factors studied, temperature has the greatest effect. Protein content is maximal at $15^{\circ} \mathrm{C}$, while lipid and carbohydrate contents were maximal at $25^{\circ} \mathrm{C}$ and $35^{\circ} \mathrm{C}$ respectively. Light intensity has no effect on protein and carbohydrate content, but the lipid component decreases by approximately $20 \%$ at a light intensity of $300 \mu \mathrm{mol}$ quanta. $\mathrm{m}^{-2} \cdot \mathrm{s}^{-1}$. Moreover the nitrogen source has no significant effect on the cellular content of these three compounds. An increase in nitrogen concentration induces an increase in protein and lipid content with, however, a reduction in biomass production above concentrations of $400 \mathrm{mg} \mathrm{l}^{-1} \mathrm{KNO}_{3}$. Modifications of the biochemical composition of Scenedesmus abundans as a function of the growth phase have also been observed. In the stationary growth phase, these modifications can be summarised as a marked reduction in protein content and, to a lesser extent, in lipid content, accompanied by a considerable accumulation of carbohydrates. The results are analysed taking into account the production yield of each of the three types of compound.

\footnotetext{
1. Equipe Biotechnologie Algale (JER 5001 associée à l'Agence Universitaire de la Francophonie "ex AUPELF-UREF"). Laboratoire de Physiologie Végétale. Faculté des Sciences Semlalia,. Avenue Prince Moulay Abdallah. B.P. 2390. Marrakech. Maroc.

2. Auteur correspondant : E-mail: belkoura@ucam.ac.ma

3. Centre d'Ecologie des Systèmes Aquatiques Continentaux, UMR C5576 CNRS, Université Paul Sabatier, 118 route de Narbonne, F-31062

Toulouse Cedex, France.
} 


\section{Introduction}

L'utilisation des microalgues à des fins appliquées (alimentation, médecine, cosmétologie, aquaculture, etc ...) s'est nettement développée depuis les années 80 (pour revue, voir Borowitzka 1995). Parmi les algues cultivées actuellement dans divers pays, les espèces du genre Scenedesmus sont exploitées dans l'alimentation humaine et animale. En effet, de par leurs caractéristiques biochimiques particulièrement intéressantes, notamment des teneurs élevées en protéines (comparables à celles des céréales), une bonne composition en acides aminés, en acides gras (en particulier en acides gras polyinsaturés) et en vitamines (y compris la vitamine $B_{12}$, déficiente chez de nombreuses plantes fourragères), ces microalgues constituent un aliment de qualité (Becker 1988, 1994). Par ailleurs, outre leur très grande diversité spécifique, la plasticité de leur métabolisme permet d'orienter ces organismes vers la production du ou des métabolites souhaités, en fonction des conditions de culture adoptées.

De nombreux systèmes de production en masse ont ainsi été développés pour diverses espèces appartenant au genre Scenedesmus: Scenedesmus obliquus (Payer et al. 1978, Becker \& Venkataraman 1982, El Fouly et al. 1985), Scenedesmus acutus (Gross et al. 1982), Scenedesmus quadricauda (Simmer 1969). Parmi cellesci, Scenedesmus obliquus demeure, jusqu'à présent, l'espèce la plus productive en culture en masse - jusqu'à $54 \mathrm{~g}$ de matière sèche $\cdot \mathrm{m}^{-2} \cdot \mathrm{d}^{-1}$ - (Soeder \& Hegewald 1988). Cependant, il convient de souligner qu'un nombre limité d'espèces de ce genre est actuellement exploité. Si l'on considère l'effectif répertorié à ce jour (200 à 250 espèces) il apparaît qu'un criblage plus exhaustif des espèces susceptibles d'avoir un intérêt alimentaire ou industriel s'impose. Ce criblage devrait cependant prendre en considération un certain nombre de critères essentiels, à savoir une croissance rapide des souches sélectionnées, une capacité d'adaptation à diverses conditions environnementales et une composition biochimique intéressante (teneur élevée en protéines ou production de substances à haute valeur ajoutée). C'est dans cette optique particulière qu'a été initié ce travail sur l'algue verte Scenedesmus abundans. Bien que cette espèce ait fait l'objet d'études antérieures (Giddings 1977, Nishijima et al. 1979), ses caractéristiques biochimiques restent à ce jour très fragmentaires. En effet, les seuls travaux réalisés concernent l'influence de la limitation en azote sur les teneurs cellulaires en carbone, azote et chlorophylle (Giddings 1977), ainsi que l'analyse de la composition en vitamines de cette espèce (Nishijima et al. 1979).
Dans le but d'évaluer les perspectives de valorisation de Scenedesmus abundans, nous avons, dans un premier temps, analysé sa composition biochimique globale. Les teneurs en protéines, glucides et lipides des cellules algales ont été évaluées à l'aide de cultures soumises à différents types de facteurs : héliothermiques - température, intensité lumineuse - nutritionnels - nature et concentration de la source azotée - physiologique - phase de croissance -.

\section{Matériel et méthodes}

\subsection{Conditions de culture}

L'algue verte Scenedesmus abundans a été isolée du lac de barrage Lalla Takerkoust (région de Marrakech, Maroc). La souche mère utilisée dans toutes les expérimentations est monoalgale mais non axénique. Cependant, son repiquage régulier dans des conditions d'asepsie et sa mise en culture dans un milieu entièrement minéral réduisent considérablement les risques de contamination bactérienne (Dauta 1982).

La souche mère, cultivée en milieu fini (batch), est maintenue en phase exponentielle de croissance par des repiquages chaque 2 à 3 jours, dans des conditions de température et d'intensité lumineuse suboptimales (i.e. $25^{\circ} \mathrm{C}, 260 \mu \mathrm{mol}$ quanta. $\mathrm{m}^{-2} . \mathrm{s}^{-1}$ ). Elle est incubée sous une photopériode de 15 heures de lumière pour 9 heures d'obscurité $(15 / 9 ; \mathrm{L} / \mathrm{O})$. Les cultures sont réalisées dans des Erlenmeyers de $500 \mathrm{ml}$ à 2 litres, stérilisés par autoclavage $\left(120^{\circ} \mathrm{C}, 20 \mathrm{~min}\right)$. Afin d'éviter la sédimentation cellulaire et de prévenir l'appauvrissement du milieu en $\mathrm{CO}_{2}$, les cultures sont fortement bullées $\left(0,51 \mathrm{~d}^{\prime}\right.$ air. $1^{-1}$ culture. $\left.\mathrm{min}^{-1}\right)$ avec de l'air comprimé filtré au travers d'une capsule de $0,2 \mu \mathrm{m}$ de porosité (Gelman). Les expérimentations sont réalisées dans une enceinte thermorégulée. La lumière est apportée par une lampe Phytoclaude de 400 watts placée à la verticale des cultures. L'intensité lumineuse incidente est mesurée à l'aide d'un quantamètre Li-Cor 185A.

Avant chaque expérience, les cultures sont préadaptées aux conditions retenues par des repiquages successifs (au moins 3 ). Elles sont ensuite (au temps $t_{0}$ de chaque expérience) largement diluées dans un milieu neuf afin d'éviter tout phénomène d'auto-ombrage, ainsi que les risques éventuels de limitation par les nutriments.

Les expériences relatives à l'influence de la température sur la composition biochimique de Scenedesmus abundans ont été réalisées sous une intensité lumineuse de $300 \mu \mathrm{mol}$ quanta. $\mathrm{m}^{-2} . \mathrm{s}^{-1}$, avec une photopériode de $15 / 9(\mathrm{~L} / \mathrm{O})$ et à 3 températures différentes $(15,25$, 
$35^{\circ} \mathrm{C}$ ). Celles destinées à évaluer l'effet de l'intensité lumineuse ont été effectuées à une température de $35^{\circ} \mathrm{C}$, sous une photopériode de $15 / 9(\mathrm{~L} / \mathrm{O})$ et sous 3 intensités lumineuses $(75,150,300 \mu \mathrm{mol}$ quanta. $\left.\mathrm{m}^{-2} \cdot \mathrm{s}^{-1}\right)$.

L'étude de l'influence de la nature de la source azotée sur la composition biochimique globale de Scenedesmus abundans a été réalisée sous les conditions héliothermiques suivantes : température $=30^{\circ} \mathrm{C}$, intensité lumineuse $=300 \mu \mathrm{mol}$ quanta. $\mathrm{m}^{-2} \cdot \mathrm{s}^{-1}$, photopériode $=15 / 9(\mathrm{~L} / \mathrm{O})$. L'azote est apporté sous forme de nitrate de potassium (constituant du milieu de culture standard), de chlorure d'ammonium ou d'urée, à la concentration de $27,69 \mathrm{mg} \mathrm{N} .1^{-1}$. Le milieu de culture est tamponné à pH 7,8 avec du Tris $2 \mathrm{mM}$, et ce afin d'éviter les variations brusques du $\mathrm{pH}$ liées à l'introduction des différentes sources d'azote. Les expérimentations relatives à l'effet des différentes concentrations en nitrate de potassium sur la composition biochimique ont été réalisées dans les conditions de culture précitées, avec des apports initiaux en $\mathrm{KNO}_{3}$ compris entre 200 et $1600 \mathrm{mg} . \mathrm{l}^{-1}$.

Pour toutes les différentes analyses, les prélèvements de la biomasse algale sont effectués en phase exponentielle de croissance (au 3 ème jour de culture), à la fin de la phase éclairée.

Les variations de la composition biochimique de Scenedesmus abundans en fonction du stade de croissance ont été évaluées à partir de cultures réalisées à $30^{\circ} \mathrm{C}, 300 \mu \mathrm{mol}$ quanta. $\mathrm{m}^{-2} . \mathrm{s}^{-1}$ et sous une photopériode de 15/9 (L/O). Les analyses ont été effectuées sur la biomasse algale prélevée en phase exponentielle (3ème jour de culture) et en phase stationnaire de croissance (12 ème jour).

Les résultats des différentes expériences correspondent à la moyenne de trois répétitions indépendantes.

\subsection{Méthodes}

La détermination de la matière sèche (MS) a été réalisée sur des échantillons de 25 à $200 \mathrm{ml}$ selon la densité de la culture. La suspension algale est filtrée sur un filtre en fibre de verre (filtre Wathman GF/C, $\varnothing 47$ $\mathrm{mm}$ ) prépesé (balance Mettler AE260, précision : $\pm 10^{-4} \mathrm{~g}$ ). Le filtre est ensuite séché dans une étuve à $80^{\circ} \mathrm{C}$ jusqu'à l'obtention d'une masse constante. La différence des pesées permet de déterminer le poids sec de l'échantillon. Les résultats correspondent à la moyenne de trois réplicats.

L'extraction des protéines a été réalisée par la technique de Rausch (1981) modifiée par l'ajout de désoxycholate de sodium (Belkoura 1994). Le dosage a ensuite été réalisé par la méthode de Bradford
(1976). Les résultats correspondent à la moyenne de neuf réplicats et sont exprimés en équivalent BSA (albumine bovine sérique).

Les glucides ont été analysés par la méthode "phénol-acide sulfurique" décrite par Kochert (1978). Les résultats, exprimés en équivalent glucose, correspondent à la moyenne de 5 répétitions.

L'extraction et le dosage des lipides ont été réalisés selon la méthode de Kates (1972).

\section{Résultats}

Les variations de la composition protéique, glucidique et lipidique de Scenedesmus abundans, cultivée sous 3 températures différentes sont représentées dans la figure 1 . L'analyse des résultats obtenus montre que la synthèse protéique évolue à la baisse avec l'accroissement de la température de culture. Inversement, la teneur en glucides passe du simple au double, entre 15 et $35^{\circ} \mathrm{C}$. Les composés lipidiques présentent, quant à eux, un maximum à la température intermédiaire de $25^{\circ} \mathrm{C}$. L'augmentation de la température du milieu de culture engendre, par ailleurs, un accroissement particulièrement important de la biomasse algale (facteur voisin de 3, Fig. 1). Cet effet se répercute positivement

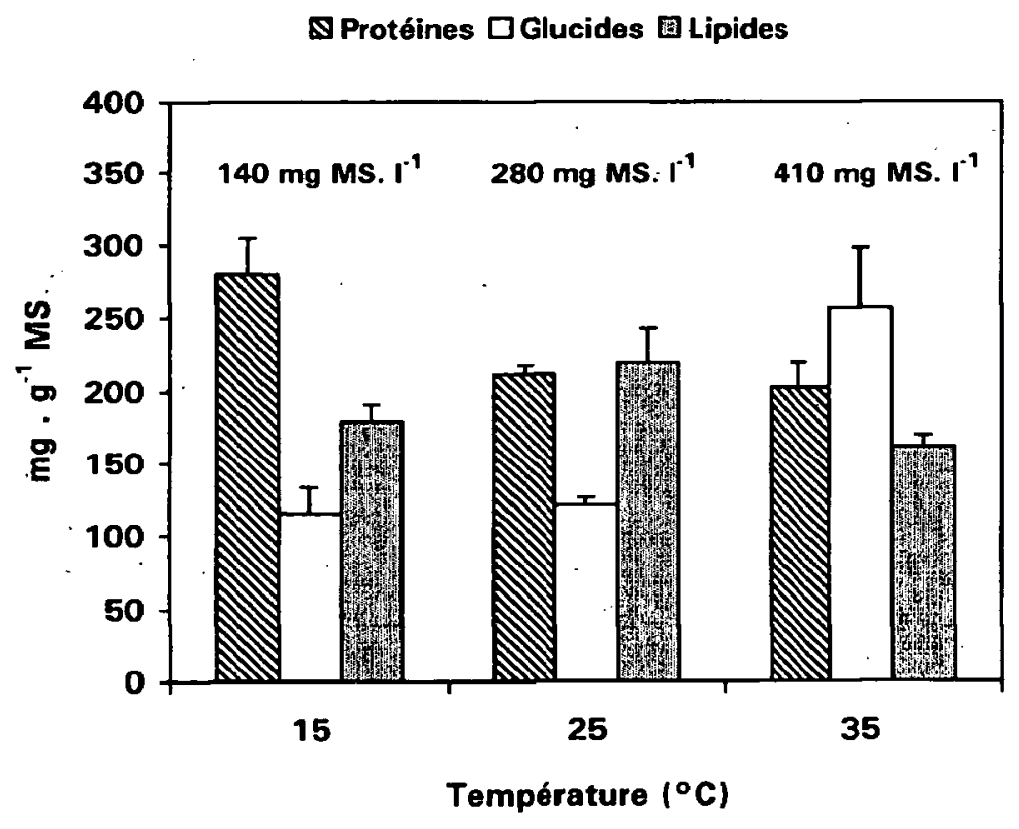

Fig. 1. Evolution de la composition biochimique de Scenedesmus abundans en fonction de la température d'incubation. (Intensité lumineuse $=300 \mu \mathrm{mol}$ quanta. $\mathrm{m}^{-2} \cdot \mathrm{s}^{-1}$. Les chiffres mentionnés dans la partie supérieure du graphique représentent la concentration de la biomasse algale. Les barres verticales indiquent l'écarttype).

Fig. 1. Changes in the biochemical composition of Scenedesmus abundans as a function of the cultivation temperature. (Light intensity $=300 \mu \mathrm{mol}$ quanta.m ${ }^{-2} \cdot \mathrm{s}^{-1}$. Numbers above the graph indicate algal biomass concentration. Vertical bars denote standard deviation). 
sur les rendements de production (rapport des teneurs des constituants et de la biomasse produite) des différents métabolites; les valeurs les plus élevées étant observées à $35^{\circ} \mathrm{C}$ (Tableau 1 ).

Parallèlement aux expérimentations précédentes, l'influence de l'intensité lumineuse sur la composition biochimique de Scenedesmus abundans a également été évaluée. Pour cela, trois éclairements d'intensité variable $\left(75,150\right.$ et $300 \mu \mathrm{mol}$ quanta. $\left.\mathrm{m}^{-2} . \mathrm{s}^{-1}\right)$ ont été retenus. Les résultats des analyses effectuées sont représentés dans la figure 2 . Ils montrent en particulier que les synthèses protéique et glucidique ne semblent pas affectées de manière très marquée par l'intensité lumineuse incidente, le rapport glyco-protéique ne subissant qu'une légère augmentation (de 1,18 à 1,27 , entre 75 et $300 \mu \mathrm{mol}$ quanta. $\mathrm{m}^{-2} \cdot \mathrm{s}^{-1}$ ). La biosynthèse des constituants lipidiques est, quant à elle, modifiée à la baisse aux fortes intensités lumineuses (réduction de $23 \%$ entre 75 et $300 \mu \mathrm{mol}$ quanta. $\mathrm{m}^{-2} . \mathrm{s}^{-1}$ ). Cependant, si l'augmentation de l'intensité lumineuse n'engendre pas de modification de la composition biochimique de l'algue, il n'en demeure pas moins qu'elle induit un accroissement substantiel de la biomasse algale (Fig. 2). En effet, les teneurs en matière sèche par litre de culture passent de 304 à $410 \mathrm{mg}$ (respectivement à 75 et 300 $\mu$ mol quanta. $\mathrm{m}^{-2} \cdot \mathrm{s}^{-1}$ ), soit une augmentation de $35 \%$. Les rendements de production des différents composés cellulaires suivent, en conséquence, la même tendance (Tableau 2).

A l'issue de ces expérimentations, nous avons donc envisagé d'optimiser la production de protéines par cette espèce, en évaluant d'une part, l'effet de la nature de la source azotée $\left(\mathrm{KNO}_{3}, \mathrm{ClNH}_{4}\right.$, urée), et d'autre part, l'influence des apports croissants en azote dans le milieu de culture. Les résultats obtenus montrent que les teneurs en protéines sont sensiblement identiques, quelle que soit la source azotée utilisée (Fig. 3). L'apport d'azote, sous forme de nitrate de potassium, produit néanmoins une algue de meilleure qualité si l'on prend en considération les trois constituants cellulaires. En outre, plus la concentration en nitrate du milieu de culture est importante, plus les teneurs en protéines sont élevées (Fig. 4). L'augmentation de la biosynthèse de ces composés est, de surcroît, accompagnée d'un accroissement de la teneur en lipides; la production de glucides ne subissant, quant à elle, que de légères variations (Fig. 4). Cependant, il faut noter que l'apport excessif de nitrate de potassium, au-delà de $400 \mathrm{mg} . \mathrm{l}^{-1}$, provoque un ralentissement de la croissance cellulaire et, par voie de conséquence, une diminution de la production de biomasse (Fig. 5). En terme de rendements, la production globale en protéines n'est
Tableau 1. Rendements de production des différents métabolites d'une culture de Scenedesmus abundans en fonction de la température (Intensité lumineuse $=\mathbf{3 0 0} \mu \mathrm{mol}$ quanta. $\mathrm{m}^{-2} \cdot \mathrm{s}^{-1}$ ).

Table 1. Yield of the different cellular compounds of the Scenedesmus abundans culture as a function of cultivation temperature (Light intensity $=300 \mu \mathrm{mol}$ quanta. $\mathrm{m}^{-2} . \mathrm{s}^{-1}$ ).

\begin{tabular}{cccc}
\hline $\begin{array}{c}\text { Température } \\
\left({ }^{\circ} \mathrm{C}\right)\end{array}$ & Protéines & $\begin{array}{c}\text { mg. } \mathbf{l}^{-1} \text { de culture } \\
\text { Glucides }\end{array}$ & Lipides \\
\hline 15 & 40 & 16 & 25 \\
25 & 59 & 34 & 62 \\
35 & 83 & 105 & 66 \\
\hline
\end{tabular}

Tableau 2. Rendements de production des différents métabolites d'une culture de Scenedesmus abundans en fonction de l'intensité lumineuse (Température $=35^{\circ} \mathrm{C}$ ).

Table 2. Yield of the different cellular compounds of the Scenedesmus abundans culture as a function of the light intensity (Temperature $=35^{\circ} \mathrm{C}$ ).

\begin{tabular}{|c|c|c|c|}
\hline $\begin{array}{l}\text { Intensité lumineuse } \\
\left(\mu \mathrm{mol} \text { quanta. } \mathrm{m}^{-2} \cdot \mathrm{s}^{-1}\right)\end{array}$ & Protéines & $\begin{array}{l}\text { mg. } .^{-1} \text { de culture } \\
\text { Glucides }\end{array}$ & Lipides \\
\hline 75 & 68 & 80 & 64 \\
\hline 150 & 76 & 89 & 63 \\
\hline 300 & 83 & 105 & 66 \\
\hline
\end{tabular}

\$ Protéines $\square$ Glucides 圆 Lipides

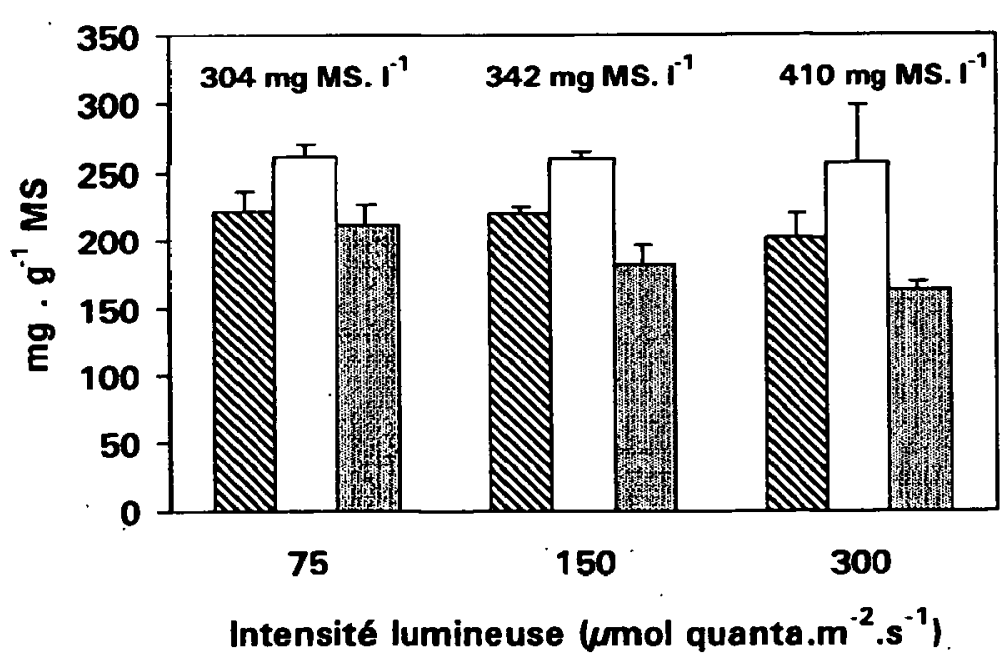

Fig. 2. Influence de l'intensité lumineuse sur la composition biochimique de Scenedesmus abundans. (Température $=35^{\circ} \mathrm{C}$. Les chiffres mentionnés dans la partie supérieure du graphique représentent la concentration de la biomasse algale. Les barres verticales indiquent l'écart-type).

Fig. 2. Effect of light intensity on the biochemical composition of Scenedesmus abundans. (Temperature $=35^{\circ} \mathrm{C}$. Numbers above the graph indicate algal biomass concentration. Vertical bars denote standard deviation). 


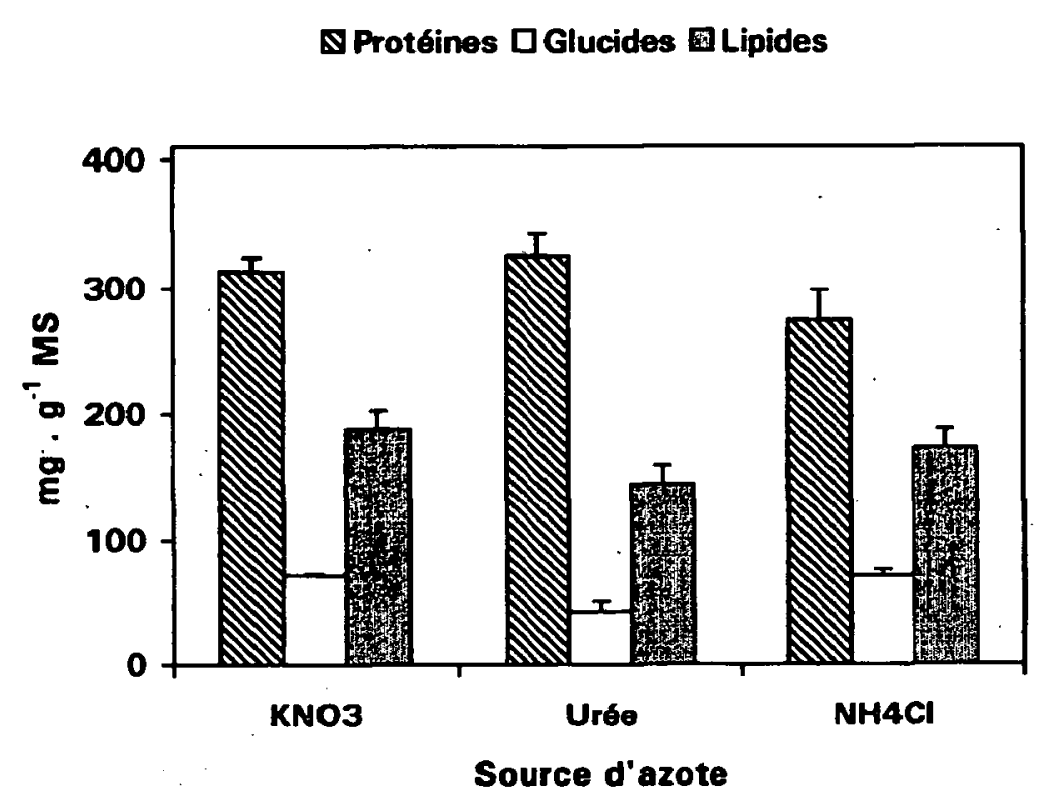

Fig. 3. Evolution de la composition biochimique de Scenedesmus abundans en fonction de la nature de la source azotée. (Température $=30^{\circ} \mathrm{C}$, Intensité lumineuse $=300 \mu \mathrm{mol}$ quanta. $\mathrm{m}^{-2} \cdot \mathrm{s}^{-1}$. Les barres verticales indiquent l'écart-type de la moyenne).

Fig. 3. Changes in the biochemical composition of Scenedesmus abundans as a function of the nitrogen source. (Temperature $=$ $30^{\circ} \mathrm{C}$, Light intensity $=300 \mu \mathrm{mol}$ quanta. $\mathrm{m}^{-2} \cdot \mathrm{s}^{-1}$. Vertical bars denote standard deviation).

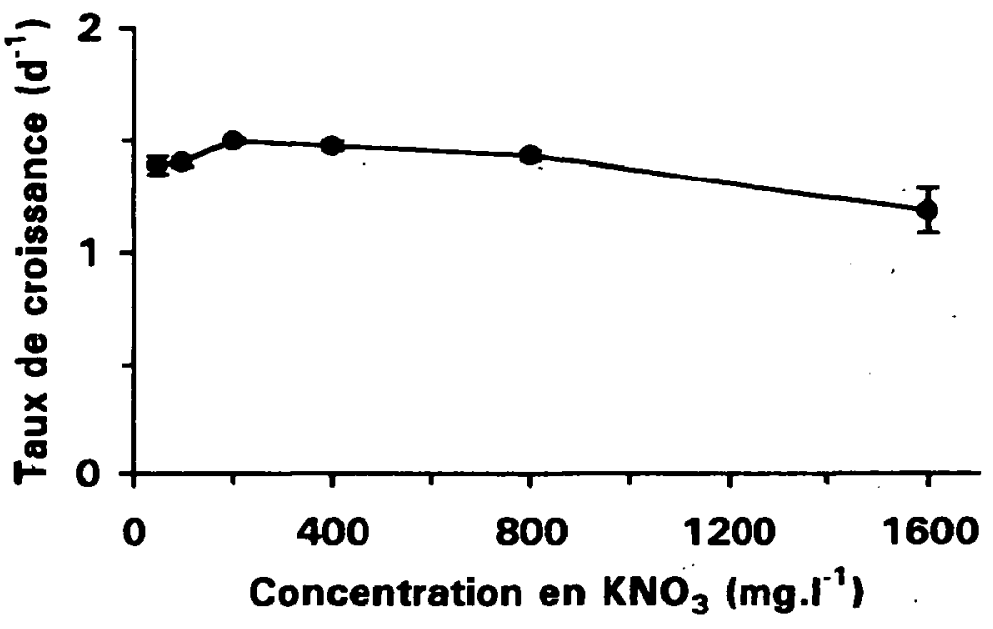

Fig. 5. Evolution du taux de croissance $(\mu)^{*}$ de Scenedesmus abundans en fonction de la concentration en nitrate du milieu de culture. . Température $=30^{\circ} \mathrm{C}$, Intensité lumineuse $=300 \mu \mathrm{mol}$ quanta. $\mathrm{mm}^{-2} \cdot \mathrm{s}^{-1}$. Les barres verticales indiquent l'écart-type de la moyenne). $* \mu=\operatorname{Ln}\left(A_{1} / A_{0}\right) * 1 / t_{1}-t_{0} ; A_{0}$ et $A_{1}$ étant les densités optiques mesurées à $750 \mathrm{~nm}$ aux temps $t_{0}$ et $t_{1}$ (temps exprimé en jour).

Fig. 5.Effect of nitrate concentration on the growth rate $(\mu)^{*}$ of Scenedesmus abundans (Temperature $=30^{\circ} \mathrm{C}$, Light intensity $=300$ $\mu \mathrm{mol}$ quanta. $\mathrm{m}^{-2} \cdot \mathrm{s}^{-1}$. Vertical bars denote standard deviation). $* \mu$ $=\operatorname{Ln}\left(A_{1} / A_{0}\right) * 1 / t_{1}-t_{0} ; A_{0}$ and $A_{1}$ are the optical densities measured at $750 \mathrm{~nm}$ in the times $t_{0}$ et $t_{1}$ (time is expressed in days).

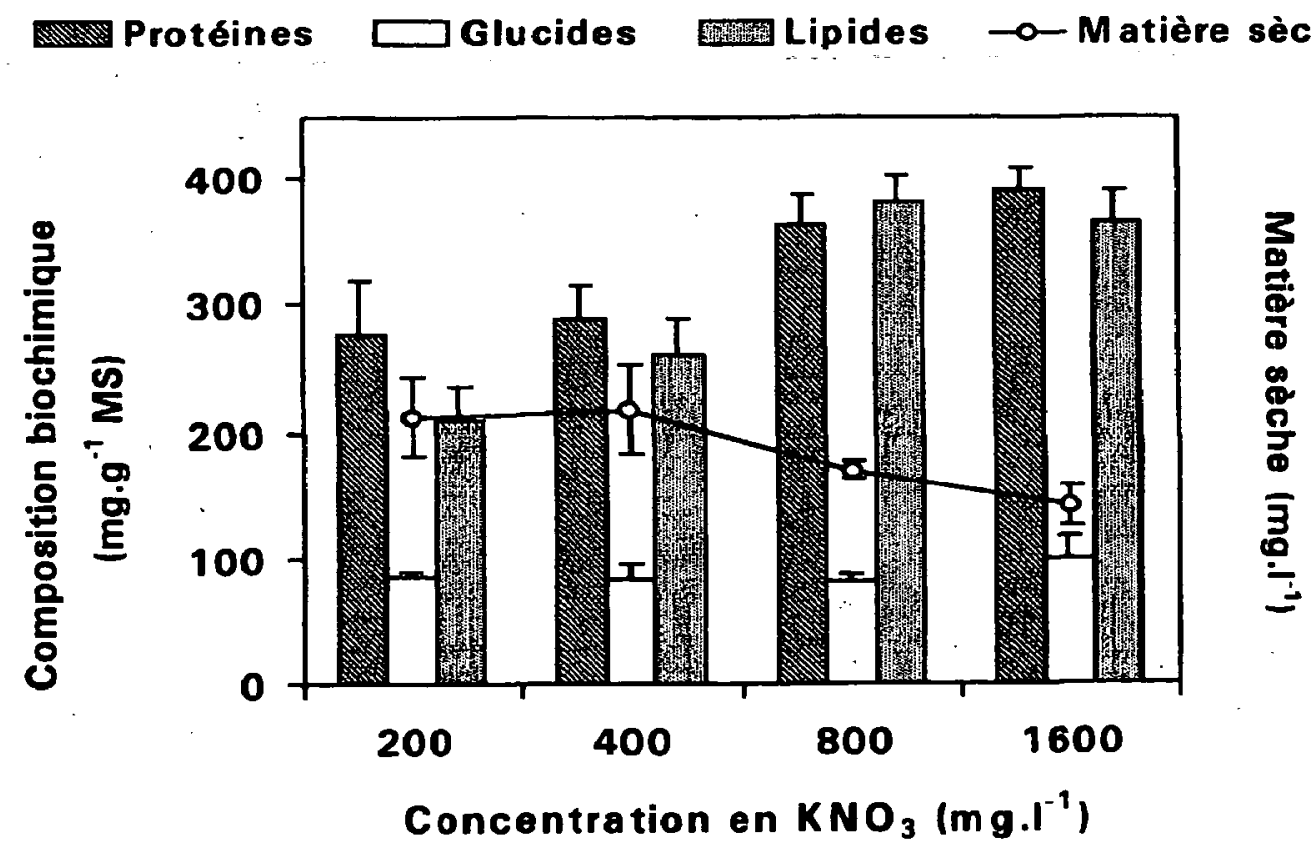

Fig. 4. Evolution de la composition biochimique de Scenedesmus abundans et de la biomasse produite en fonction de la concentration en nitrate du milieu de culture. (Température = $30^{\circ} \mathrm{C}$, Intensite lumineuse $=300 \mu \mathrm{mol}$ quanta. $\mathrm{m}^{-2} . \mathrm{s}^{-1}$. Les barres verticales indiquent l'écart-type).

Fig. 4. Changes in the biochemical composition of Scenedesmus abundans and biomass production as a function of the nitrate concentration. (Temperature $=30^{\circ} \mathrm{C}$, Light intensity $=$ $300 \mu \mathrm{mol}$ quanta. $\mathrm{m}^{-2} \cdot \mathrm{s}^{-1}$. Vertical bars denote standard deviation). 
effectivement pas améliorée de manière significative (Tableau 3).

Au-delà de l'influence qu'exercent les facteurs environnementaux sur la composition biochimique de Scenedesmus abundans, des modifications qualitatives de la biomasse algale peuvent également être observées en fonction du stade physiologique de croissance. Les analyses effectuées sur la biomasse algale prélevée au 3 ème (phase exponentielle) et au 12 2 eme jour (phase stationnaire) de culture montrent, en effet, une réduction très importante des teneurs en protéines et en lipides, avec une amplitude avoisinant 70 et $45 \%$ respectivement (Fig. $6)$. Inversement, les teneurs des composés glucidiques enregistrent un accroissement considérable $(+93 \%)$. I faut noter néanmoins que la réduction des composés protéiques et lipidiques observée en phase stationnaire est compensée par une production de biomasse 4 à 5 fois plus importante par rapport à celle élaborée en phase exponentielle; il en résulte ainsi des rendements de production plus élevés (Tableau 4).

\section{Discussion \& conclusion}

L'exploitation de la biomasse algale à des fins alimentaires ou pour la production de divers biocomposés suppose la connaissance des caractéristiques quali-

\section{QProtéines $\square$ Glucides : Lipides}

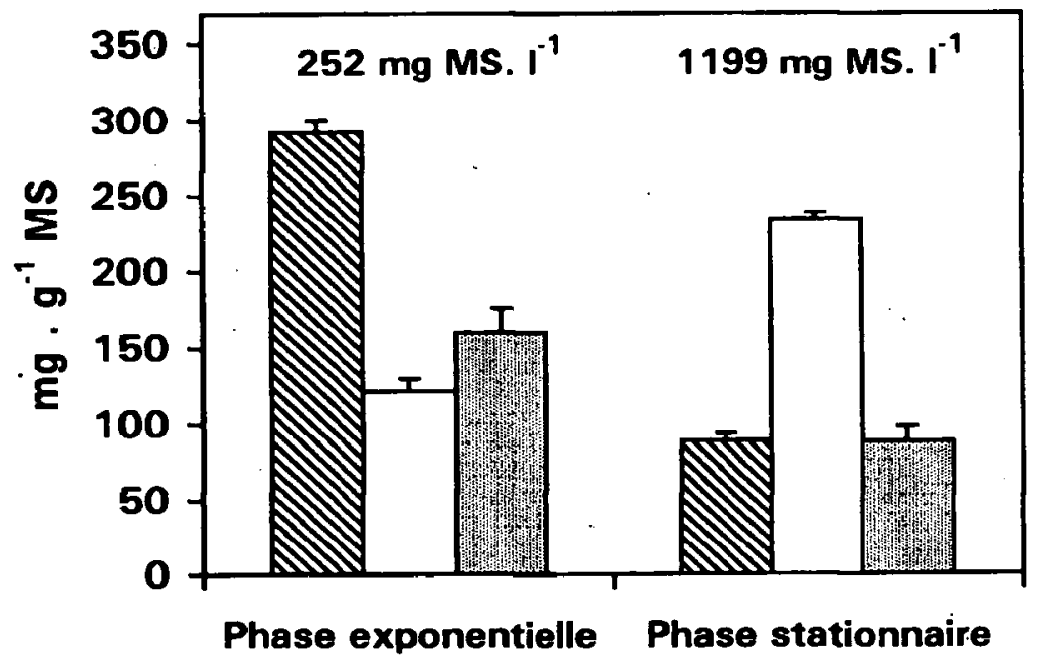

Fig. 6. Evolution de la composition biochimique de Scenedesmus abundans en fonction de la phase de croissance. (Température $=$ $30^{\circ} \mathrm{C}$, Intensité lumineuse $=300 \mu \mathrm{mol}$ quanta $\cdot \mathrm{m}^{-2} \cdot \mathrm{s}^{-1}$. Les chiffres mentionnés sur la partie supérieure du graphique représentent la concentration de la biomasse algale. Les barres verticales indiquent l'écart-type).

Fig. 6. Changes in biochemical composition of Scenedesmus abundans as a function of the growth phase. (Temperature $=30^{\circ} \mathrm{C}$, Light intensity $=300 \mu \mathrm{mol}$ quanta. $\mathrm{m}^{-2} \cdot \mathrm{s}^{-1}$. The numbers appeared in the graph indicate the algal biomass concentration. Vertical bars denote the standard deviation).
Tableau 3. Rendements de production des différents métabolites d'une culture de Scenedesmus abundans en fonction de la concentration en $\mathrm{KNO}_{3}$ du milieu de culture (Température $=$ $30^{\circ} \mathrm{C}$, Intensité lumineuse $=300 \mu \mathrm{mol}$ quanta $\cdot \mathrm{m}^{-2} \cdot \mathrm{s}^{-1}$.).

Table 3. Yield of the different cellular compounds of the Scenedesmus abundans culture as a function of the nitrate concentration (Temperature $=30^{\circ} \mathrm{C}$, Light intensity $=300 \mu \mathrm{mol}$ quanta. $\mathrm{m}^{-2} \cdot \mathrm{s}^{-1}$.).

\begin{tabular}{cccc}
\hline $\begin{array}{ccc}{\left[\mathrm{KNO}_{3}\right]} \\
\left(\mathrm{mg}^{-1}\right)\end{array}$ & Protéines & $\begin{array}{c}\text { mg.1-1 de culture } \\
\text { Glucides }\end{array}$ & Lipides \\
\hline 200 & 59 & 18 & 44 \\
400 & 63 & 18 & 57 \\
800 & 62 & 14 & 65 \\
1600 & 55 & 14 & 52 \\
\hline
\end{tabular}

Tableau 4. Rendements de production des différents métabolites d'une culture de Scenedesmus abundans en phases exponentielle et stationnaire de croissance (Température $=30^{\circ} \mathrm{C}$, Intensité lumineuse $=300 \mu \mathrm{mol}$ quanta $\mathrm{m}^{-2} \cdot \mathrm{s}^{-1}$.).

Table 4. Yield of the different cellular compounds of the Scenedesmus abundans culture as a function of the growth phase (Temperature $=30^{\circ} \mathrm{C}$, Light intensity $=300 \mu \mathrm{mol}$ quanta. $\mathrm{m}^{-2} . \mathrm{s}^{-1}$.).

\begin{tabular}{lccc}
\hline & & mg.1 le culture $^{-1}$ de & Lipides \\
\hline Phase exponentielle & 74 & 30 & 40 \\
Phase stationnaire & 107 & 281 & 106 \\
\hline
\end{tabular}

tatives de l'algue en question et une maîtrise des conditions de culture. En effet, au-delà des spécificités intrinsèques de chaque espèce, le métabolisme de ces organismes est sous la dépendance d'une multitude de paramètres environnementaux. Les résultats obtenus dans cette étude montrent que les teneurs des principaux constituants cellulaires varient, en fonction des conditions de cultures testées, entre 9 et $39 \%$ (par rapport à la matière sèche) pour les protéines, 4 et $26 \%$ pour les glucides, 9 et $36 \%$ pour des composés lipidiques. Comparé aux autres espèces du même genre, Scenedesmus abundans se caractérise par des teneurs en lipides et en glucides relativement plus élevées et un contenu en protéines sensiblement inférieur (Tableau 5). Cependant, il est important de noter que les données relatives aux teneurs en protéines sont déterminées, dans la plupart des travaux cités, à partir de l'analyse de l'azote total auquel est affecté un facteur de 6,25. Cette procédure a récemment été critiquée par de nombreux auteurs en raison de la variabilité de ce facteur selon les espèces considérées (4,97 d'après Ahlgren et al. 1992, entre 3,60 et 4,99 selon Lourenco et al. (1998) et de la prise en compte de l'azote non protéique qui peut représenter jusqu'à $20 \%$ de l'azote total 
(Becker 1988). Celle-ci aboutit, de ce fait, à une surestimation du contenu en protéines des algues. Par ailleurs, au-delà de ces considérations méthodologiques, il convient de souligner que les comparaisons interspécifiques demeurent souvent difficiles en raison de la diversité des conditions de culture. En effet, de nombreux travaux, réalisés sur des algues d'eau douce ou marines, ont montré l'influence déterminante des conditions environnementales sur la composition biochimique globale (Morris et al. 1974, Piorreck et al. 1984, Harrison et al. 1990, Renaud et al. 1991, Thompson et al. 1992, Chu et al. 1993, Aidar et al. 1994, Herrero et al. 1994, Belkoura et al. 1997, Zhu et al. 1997, Vargas et al. 1998).

Parmi les facteurs environnementaux retenus dans cette étude, la température semble avoir le plus de répercussion sur la composition biochimique de Scenedesmus abundans. Ainsi, selon les conditions thermiques du milieu, la biomasse algale présente des caractéristiques qualitatives différentes. La production de protéines est maximale à faible température $\left(15^{\circ} \mathrm{C}\right)$, alors que celle des composés lipidiques et glucidiques l'est à des températures nettement plus élevées ( 25 et $35^{\circ} \mathrm{C}$ respectivement). L'accroissement des teneurs en protéines, aux basses températures, a également été signalé dans d'autres travaux (Falkowski 1977, Piquemal 1990, Thompson et al. 1992). Ce phénomène serait étroitement lié à l'augmentation de l'incorporation du carbone dans les protéines, induite par la diminution de la température (Morris et al. 1974, Thompson et al. 1992). D'après Chapman et al. (1983) ce type de réponse refléterait les besoins croissants en enzymes des cellules ou un effet de compensation de la réduction de la fluidité membranaire.
Contraurement aux composés protéiques, les teneurs en glucides augmentent de manière considérable avec l'accroissement de la température de culture, en particulier entre 25 et $35^{\circ} \mathrm{C}$. Des observations similaires ont été effectuées sur d'autres espèces (Morris et al. 1974, Dermoun 1987, Piquemal 1990, Belkoura et al. 1997) mais il semblerait que ce résultat ne soit pas généralisable. En effet, Thompson et al. (1992) ont montré l'existence d'une diversité de comportement des espèces d'algues avec l'accroissement de la température. Chez Chaetoceros simplex, aucune variation significative n'est observée entre 10 et $25^{\circ} \mathrm{C}$, alors que chez d'autres espèces (Pavlova lutheri, Dunaliella tertiolecta, Chaetoceros calcitrans, etc...) les teneurs en glucides évoluent en "dents de scie" avec, chez certaines (Chaetoceros gracilis), une tendance à la baisse aux températures élevées. Cette dernière observation a également été rapportée par Zhu et al. (1997) chez l'algue Isochrysis galbana, dans l'intervalle de température $15-30^{\circ} \mathrm{C}$.

Par opposition aux deux premiers constituants cellulaires, l'influence de la température sur les teneurs en lipides de Scenedesmus abundans ne fait apparaître aucune tendance précise ; les pourcentages les plus élevés étant observés à une température intermédiaire $\left(25^{\circ} \mathrm{C}\right)$. Des comportements similaires ont été signalés chez d'autres espèces d'algues (Tedesco \& Duerr 1989, Tan \& Johns 1991, Thompson et al. 1992) sans qu'aucune explication physiologique ne soit avancée. En effet, une revue plus large des différents travaux réalisés sur les lipides chez les algues permet de se rendre compte de la complexité des mécanismes de régulation du métabolisme de ces composés.

Tableau 5. Données de la littérature relatives à la composition en protéines, glucides et lipides chez différentes espèces du genre Scenedesmus.

Talbeau 5. Bibliographie data for protein, carbohydrate and lipid contents in the Scenedesmus spp.

\begin{tabular}{lcccl}
\hline \multirow{2}{*}{ Espèces } & \multicolumn{3}{c}{ \% de la matière sèche } & \\
& Protéines & Glucides & Lipides & Références . \\
\hline Sc. abundans & $9-39$ & $4-26$ & $9-36$ & Présent travail \\
Sc. acutus & $27-32$ & - & 11 & Ahlgren et al. 1992 \\
Sc. dimorphus & 50 & 6 & 23 & Renaud et al.. 1994 \\
Sc. incrassatulus & $39-53$ & $12-28$ & $15-21$ & Fournadzieva et al. 1993 \\
Sc. obliquus & $45-55$ & $10-15$ & $8-12$ & Becker \& Venkatamaran 1982 \\
Sc. obliquus & $50-54$ & $11-15$ & $7-9$ & El-Fouly et al. 1985 \\
Sc. obliquus & $50-56$ & $10-17$ & $12-14$ & Payer et al: 1980 \\
Sc. obliquus & $8-34$ & - & $21-50$ & Piorreck et al. 1984 \\
Sc. obliquus & $50-56$ & $10-17$ & $12-14$ & Soeder 1981 \\
Sc. quadricauda & $23-26$ & - & $5-10$ & Ahlgren et al. 1992 \\
Sc. quadricauda & 54 & 5 & 20 & Renaud et al. 1994 \\
\hline
\end{tabular}


Parallèlement aux effets de la température du milieu de culture, nous avons également tenté d'apprécier l'influence de l'intensité lumineuse sur la composition biochimique de Scenedesmus abundans. Les résultats obtenus, dans l'intervalle d'éclairement retenu (75 à $300 \mu \mathrm{mol}$ quanta. $\mathrm{m}^{-2} \cdot \mathrm{s}^{-1}$ ), ne font apparaître aucune différence significative au niveau des teneurs en protéines et en glucides. A l'inverse, les composés lipidiques enregistrent une baisse d'environ $23 \%$ lorsque les cultures sont exposées à un éclairement élevé (300 $\mu \mathrm{mol}$ quanta. $\mathrm{m}^{-2} \cdot \mathrm{s}^{-1}$ ). L'absence d'effet de l'intensité de l'éclairement sur les teneurs en protéines a été signalé à maintes reprises dans la littérature et semble une réponse commune à de nombreuses espèces d'algues (Sukenik et al. 1989, Renaud et al. 1991, Mouget et al. 1995, Belkoura et al. 1997). Cependant, dans le cas des deux autres types de composés cellulaires (lipides et glucides), les réponses à l'accroissement de l'intensité lumineuse sont diverses (Morris et al. 1974, Dermoun 1987, Tedesco \& Duerr 1989, Harrison et al. 1990, Renaud et al. 1991, Mouget et al. 1995, Belkoura et al. 1997, Gordillo et al. 1998). Elles dénotent l'existence de spécificités intrinsèques ou d'une certaine variabilité dans la capacité d'adaptation aux différents éclairements. Il est à signaler, en outre, que les différences spectrales des sources lumineuses utilisées dans les travaux cités peuvent également être à l'origine des variations enregistrées. En effet, de nombreuses études ont montré que la qualité de la lumière avait des répercussions sur la composition biochimique des algues (Wallen \& Geen 1971, Kowallik 1978, Falkowski \& LaRoche 1991, Aidar et al. 1994).

Outre le contrôle qu'exercent les facteurs héliothermiques sur la composition biochimique des algues, celle-ci est également influencée par les conditions nutritionnelles, notamment par la nature et la concentration de la source azotée. Les résultats obtenus chez Scenedesmus abundans, montrent que la teneur en protéines n'est pas affectée de manière significative par la nature de la source azotée utilisée. Cependant, sur le plan qualitatif, les algues cultivées en présence de $\mathrm{KNO}_{3}$ présentent une meilleure composition biochimique globale. Ces observations sont en accord avec les travaux de Piorreck et al. (1984) sur Chlorella vulgaris et Scenedesmus obliquus, bien que les effets des différentes sources d'azote n'aient pas été dissociés des variations de $\mathrm{pH}$ qu'elles induisent. Il est actuellement bien établi que l'absorption des ions $\mathrm{NO}_{3}$ - induit une alcalinisation du milieu de culture alors que celle des ions $\mathrm{NH}_{3}$ entraîne, au contraire, une acidification de celui-ci (Goldman \& Brewer 1980). Ainsi, en milieu non tamponné, les observations effectuées reflètent à la fois les effets du pH sur la croissance et/ou le degré d'efficacité des différentes formes azotées. Dans les expérimentations que nous avons réalisées sur Scenedesmus abundans, l'utilisation du Tris (Cf $\S 2.1$.) a permis de pallier ce problème. D'une manière générale, des études récentes ont montré que les variations de la composition biochimique sont plus ou moins spécifiques et dépendent de la capacité de chaque espèce à se développer sur tel ou tel type de source azotée (Soliman 1993, Ionescu et al. 1994, Lourenco et al. 1997, Fidalgo et al. 1998). Par ailleurs, l'accroissement de la concentration en azote dans le milieu de culture induit une amélioration de la composition biochimique globale de Scenedesmus abundans (augmentation des teneurs en protéines et en lipides), avec toutefois une réduction de la production de biomasse pour des concentrations en $\mathrm{KNO}_{3}$ supérieures à $400 \mathrm{mg} \cdot \mathrm{l}^{-1}$. L'existence d'une corrélation positive entre la concentration en azote et les teneurs en protéines n'est plus à démontrer (Piorreck et al. 1984, Herrero et al. 1994, Gordillo et al. 1999). Cependant, si cette réponse semble être la règle générale pour la majorité des algues étudiées, il n'en est pas de même des composés lipidiques dans le cas pour lesquels les effets de la concentration en azote sont spécifiques. Ainsi, chez Spirulina platensis (Piorreck et al. 1984, Gordillo et al. 1999), Hymenomonas carterae (Shifrin \& Chisholm 1981), Microcystis aeruginosa (Piorreck et al. 1984) et Dunaliella tertiolecta (Herrero et al. 1994), une accumulation des composés lipidiques est consécutive à l'enrichissement en azote du milieu de culture. Le résultat inverse est obtenu chez d'autres espèces : Chlamydomonas applanata et Chlorella pyrénoidosa (Shifrin \& Chisholm 1981), Chlorella vulgaris et Scenedesmus obliquus (Piorreck et al. 1984). Concernant l'influence des apports croissants en azote sur l'accumulation de substances glucidiques, nos résultats n'indiquent aucun effet significatif : il en est de même chez Dunaliella tertiolecta (Herrero et al. 1994). Cependant, Harrison et al. (1990) ainsi que Gordillo et al. (1999) rapportent une tendance à la hausse lorsque les cultures sont appauvries en azote.

Au-delà des variations induites par les différentes conditions environnementales retenues dans cette étude, nous avons également mis en évidence des modifications de la composition biochimique de Scenedesmus abundans en fonction du stade de croissance. En phase de stationnaire croissance, ces modifications se résument en une réduction marquée des teneurs en protéines et, dans une moindre mesure, en lipides, avec par contre une forte accumulation des composés glucidiques. Des observations similaires ont été rapportées à maintes reprises chez diverses espèces (Chu et al. 1993, Herrero et al. 1994, Belkoura et al. 1997, Zhu et 
al. 1997). Trois facteurs essentiels peuvent expliquer cette évolution, 1) l'état physiologique des cellules, 2) la réduction de la lumière disponible par effet d'autoombrage, 3) la déficience en nutriments, et en particulier, en azote. Dans les cultures en "batch", il est très difficile, sinon impossible, de dissocier les effets de ces trois facteurs. Néanmoins, les variations de la composition biochimique observées en phase stationnaire de croissance retracent parfaitement celles obtenues dans les expériences de limitation en azote (résultats non publiés). Ceci laisse supposer que le déficit en azote du milieu de culture semble bien le facteur prépondérant dans cette évolution.

Dans un but de valorisation de la biomasse algale, la connaissance de la composition biochimique de l'espèce et des variations induites par les conditions environnementales sont d'une importance primordiale dans les programmes de criblage. Cependant, les données exprimées en valeurs relatives (mg. ${ }^{-1}$ de MS notamment) ne rendent pas toujours compte de la production globale d'une culture. L'évaluation des rendements de production d'une substance donnée, intégrant la quantité totale de biomasse élaborée, est également un facteur déterminant. Dans le présent travail, nous avons montré effectivement que cette notion de rendement avait toute sa signification et qu'elle devait être prise en compte pour le choix des conditions de culture. A titre d'exemple, les teneurs maximales en protéines chez Scenedesmus abundans ont été enregistrées à $15^{\circ} \mathrm{C}$ (Fig. 1), alors qu'en terme de rendement de la culture, c'est à $35^{\circ} \mathrm{C}$ que la production est optimale (Tableau 1).

En définitive, l'étude réalisée chez Scenedesmus abundans a permis de caractériser cette espèce sur le plan biochimique et d'évaluer l'impact des paramètres de culture sur les teneurs de ses principaux constituants cellulaires. Bien qu'elle ne soit pas particulièrement riche en protéines, les travaux en cours de réalisation semblent indiquer que cette algue recèle d'autres caractéristiques intéressantes, notamment la production de caroténoides secondaires en situation de carence azotée et de substances à activité antibactérienne.

\section{Remerciements}

Ce travail a été soutenu par l'Agence Universitaire de la Francophonie (A.U.F.) dans le cadre d'un contrat d'association "JER 5001" du Fond Francophone de la Recherche (FFR). Les auteurs remercient cet organisme pour son soutien financier.

L'identification de l'espèce d'algue utilisée dans cette étude a été réalisée par le Professeur M. Loudiki Responsable du Laboratoire d'Algologie de la Faculté des Sciences Semlalia, que nous tenons à remercier.

Les auteurs rermercient les lecteurs (référés) pour les critiques qui ont permis l'amélioration du manuscrit.

\section{Travaux cités}

Ahlgren G., Gustafsson I.B. \& Boberg M. 1992. - Fatty acid content and chemical composition of freshwater microalgae. $J$. Phycol., 28 : 37-50.

Aidar E., Gianesella-Galvao S.M.F., Sigaud T.C.S., Asano C.S., Liang T.H., Rezende K.R.V., Oishi M.K., Aranha F.J., Milani G.M. \& Sandes M.A.L. 1994. - Effects of light quality on growth, biochemical composition and photosynthesis production in $C y$ clotella caspia Grunow and Tetraselmis gracilis (Kylin) Butcher. J. of Experimental Marine Biology and Ecology, 180 : 175-187.

Becker E.W. 1988. - Micro-algae for human and animal consumption. In Micro-algal Biotechnology. Borowitzka M.A. \& Borowitzka L.J., (Eds.), Cambridge University Press, London : 222-256.

Becker E.W. 1994. - Microalgae : Biotechnology and Microbiology. Baddiley J., Carey N.H., Higgins I.J. \& Potter W.G. (Eds.), Cambridge Studies in Biotechnology, Cambridge : $293 \mathrm{p}$.

Becker E.W. \& Venkatamaran L.V. 1982. - Biotechnology and Exploitation of Algae : The Indian Approach. Eschborn : German Agency for Technical Cooperation GmbH.

Belkoura M. 1994. - Chlorella sorokininana Shihira \& Krauss: Ecophysiologie, modélisation de cultures et perspectives de valorisation de la biomasse algale. Thèse de Doctorat d'Etat, Université Cadi Ayyad, Faculté des Sciences Semlalia : 154 p. (+annexe).

Belkoura M., Benider A. \& Dauta A. 1997. - Influence de.la température, de l'intensité lumineuse et du stade de croissance sur la composition biochimique de Chlorella sorokiniana Shihira \& Krauss. Annls Limnol., 33 (1) : 3-11.

Borowitzka M.A. 1995. - Microalgae as sources of pharmaceuticals and other biologically active compound. J. applied.Phycol., $7: 3-15$.

Bradford M.M. 1976. - A rapid and sensitive method for the quantitation of microgram quantities of protein utilizing the principle of protein-dye binding. Analyt. Biochem., 72 : 248-254.

Chapman D.J., DeFelice J. \& Barber J. 1983. — Growth temperature effects on thylakoid membrane lipid and protein content of pea chloroplasts. Plant Physiol. (Bethesda), 72 : 225-228.

Chu W-L., Phang S-M. \& Goh S-H. 1993. - Variations in growth, biochemical composition and pigmentation of Ankistrodesmus convolutus cultured under 12:12 $\mathrm{H}$ light-dark cycle and continuous illumination. In $6^{\text {th }}$ International Conference on Applied Algology. Ceske Budejovice, République Tchèque, 6-11 Septembre 1993.

Dauta A. 1982. - Conditions de développement du phytoplancton. Etude comparative de huit espèces en culture. I. Détermination des paramètres de croissance en fonction de la lumière et de la température. Annls Limnol., 18 (3) : 217-262.

Dermoun D. 1987. - Ecophysiologie de Porphyridium cruentum : validation expérimentale d'un modèle de croissance. Etude de la production de polysaccharide. Thèse de Doctorat, Université de Technologie de Compiègne, France : $211 \mathrm{p}$.

El-Fouly M.M., Mohn F.H. \& Soeder C.J. 1985. - Joint EgyptianGerman project. Intensive protein production through microalgae. Kernforschungsanlage Jülich GmbH, Publication, 34/85.1.

Falkowski P.G. 1977. - The adenylate energy charge in marine phytoplankton : the effect of temperature on the physiological state of Skeletonema costatum (Cleve.) Cleve. J. Exp. Mar. Biol. Ecol., 27 : 37-45.

Falkowski P.G. \& LaRoche J. 1991. - Acclimatation to spectral irradiance in algae. J. Phycol., $27: 8-14$.

Fidàlgo J.P., Cid A., Torres E., Sukenik A. \& Herrero C. 1998. - Effects of nitrogen source and growth phase on proximate biochemical composition, lipid classes and fatty acid profile of the marine microalga Isochrysis galbana. Aquaculture, 166(1-2) : 105-116. 
Fournadzieva S., Gabev A., Pilarski P. \& Dittrt F. 1993. - Oxygen evolution, productivity and biomass quality of open mass algal culture under condition of increased medium bicarbonate content. Algological Studies, 71 : 103-110.

Giddings J.M. 1977. - Chemical composition and productivity of Scenedesmus abundans in nitrogen limited chemostat cultures. Limnol. Oceanogr., 22 (5) : 911-918.

Goldman J.C. \& Brewer P.G. 1980. — Effect of nitrogen source and growth rate on phytoplankton-mediated changes in alkalinity. Limnol. Oceanogr., 25 : 352-357.

Gordillo F.J.L., Goutx M., Figueroa F.L. \& Niell F.X. 1998. - Effects of light intensity, $\mathrm{CO}_{2}$ and nitrogen supply on lipid class composition of Dunaliella viridis. J. applied Phycol., 10 : 135-144.

Gordillo F.J.L., Jiménez C., Figueroa F.L. \& Niell F.X. 1999. - Effects of increased atmospheric $\mathrm{CO}_{2}$ and $\mathrm{N}$ supply on photosynthesis, growth and cell composition of the cyanobacterium Spirulina platensis (Arthospira). J. applied Phycol., $10: 461-469$.

Gross R., Schöneberger H., Gross U. \& Lorenzen H. 1982. - The nutritional quality of Scenedesmus acutus produced in a semiindustrial plant in Peru. Berichte der Deutschen Botanischen Gesellschaft, $95: 323-327$.

Harrison P.J., Thompson P.A. \& Calderwood G.S. 1990. — Effects of nutrient and light limitation on the biochemical composition of phytoplankton. J. applied Phycol., 2 : 45-56.

Herrero C., Abalde J., Cid A., Fidalgo P. \& Fabregas J. 1994. - Variations in the biochemical profile of the marine microalga Dunaliella tertiolecta (Butcher) cultured with different nutrient concentrations and urea as a nitrogen source. Cah. Biol. Mar., $35: 213-223$.

Ionescu A., Stanca D. \& Vladimirescu A. 1994. - The growth of some green alga (Scenedesmus, Chlorella, Botryococcus, Ulva) : physiology and biochemistry. Biologia, Bratislava, 49/4 : 605-610.

Kates M. 1972. - Techniques of lipidology. Isolation, analysis and identification of lipids. Work T.S. \& Work E. (Eds.), North-Holland Publishing Company, Amsterdam : $610 \mathrm{p}$.

Kochert G. 1978. - Carbohydrate determination by the phenolsulfuric acid method. In Handbook of Phycological Methods. Physiological and Biochemical Methods. Hellebust J. \& Craigie J., (Eds.), Cambridge University Press, London : 95-98.

Kowallik W. 1978. - Blue light effects on carbohydrate and protein metabolism. In Blue Light Responses : Phenomena and Occurrence in Plants. Senger H., (Ed.),CRC Press, Florida, Vol. 1 : 8-13.

Lourenço S.O., Barbarino E., Lanfer Marquez U.M. \& Aidar E. 1998. - Distribution of intracellular nitrogen in marine microalgae : basis for the calculation of specific nitrogen-to-protein conversion factors. J. Phycol., 34 : 798-811.

Lourenço S.O., Barbarino E., Mancini-Filho J., Shinke K.P. \& Aidar E. 1997. - Biochemical composition and growth of marine microalgae under different nitrogen source. Phycologia, 36 (4) : 66.

Morris I., Glover E. \& Yentsch C.S. 1974. - Products of photosynthesis by marine phytoplankton : the effect of environmental factors on the relative rates of protein and carbohydrate synthesis. Mar. Biol., 27 : 1-9.

Mouget J.L., Legendre L. \& de la Noüe J. 1995. — Long-term acclimatization of Scenedesmus bicellularis to high-frequency intermittent lighting $(100 \mathrm{~Hz})$. II. Photosynthetic pigments, carboxylating enzymes and biochemical composition. J. Plankton Research, 17 (4) : 875-890.

Nishijima T., Shiozaki R. \& Hata Y. 1979. - Production of vitamin $B_{12}$, thiamine and biotin by freshwater phytoplankton. Bulletin of the Japanese Society for Scientific Fisheries, 45 : 199-204.
Payer H.D., Pabst W. \& Runkel K.W. 1980. - Review of the nutrient and toxicological properties of the green algae Scenedesmus obliquus as a single-cell protein. In Algae Biomass. Shelef G. \& Soeder C.J., (Eds.), Elsevier Press, Amsterdam : 787-798.

Payer H.D., Pithakpol B., Nguitragool M., Prabharaksa C., Thananunkul D. \& Chavana S. 1978. - Major results of the Thai-German Microalgae Project at Bangkok. Archiv für Hydrobiol., Ergebnisse der Limnologie, 11 : 41-55.

Piorreck M., Baasch K.H. \& Pohl P. 1984. - Biomass, production, total protein, chlorophylls, lipids and fatty acids of freshwater green and blue-green algae under different nitrogen regimes. Phytochemistry, 23 (2) : 207-216.

Piquemal F. 1990. - Contribution à l'étude écophysiologique de quelques espèces d'algues en culture. Thèse de Doctorat, Université Paul Sabatier, Toulouse III : $137 \mathrm{p}$.

Rausch T. 1981. - The estimation of micro-algal protein content and its meaning to the evaluation of algal biomass. I. Comparison of methods for extracting protein. Hydrobiologia, $78: 237-251$.

Renaud S.M., Parry D.L. \& Thinh L.V. 1994. - Microalgae for use in tropical aquaculture I : Gross chemical and fatty acid composition of twelve species of microalgae from the Northern Territory, Australia. J. applied Phycol., 6 : 337-345.

Renaud S.M., Parry D.L., Thinh L.V., Kuo C., Padovan A. \& Sammy N. 1991. - Effect of light intensity on the proximate biochemical and fatty acid composition of Isochrysis sp. and Nannochloropsis oculata for use in tropical aquaculture. J. applied Phycol., $3:$ 43-53.

Shifrin N.S. \& Chisholm S.W, 1981. — Phytoplankton lipids : interspecific differences and effects of nitrate, silicate and light-dark cycles. J. Phycol., $17:$ 374-384.

Simmer J. 1969. - Outdoor mass cultivation of Scenedesmus quadricauda (Turp.) Breb. in South Bohemia. In Studies in Phycology. Fott B., (Ed.), E. Schweitzerbartsche Verlagsbuchhandlung, Stuttgart : 293-304.

Soeder C.J. 1981. - Chemical composition of microalgal biomass as compared to some other types of single-cell protein (SCP). University of the Orange Free State, Publications Series C, 3 : 73-85.

Soeder C.J. \& Hegewald E. 1988. - Scenedesmus. In Micro-algal Biotechnology. Borowitzka M.A. \& Borowitzka L.J., (Eds.), Cambridge University Press, London : 59-84.

Soliman A-R. I. 1993. - Effect of urea and glutamic acid on the growth and protein production of Scenedesmus quadricauda. Phykos, 32 (1\&2) : 125-130.

Sukenik A., Carmeli Y. \& Berner T. 1989. - Regulation of fatty acid composition by irradiance level in the Eustigmatophyte Nannochloropsis sp. J. Phycol., 25 : 686-692.

Tan C.K. \& Johns M.R. 1991. - Fatty acid production by heterotrophic Chlorella saccharophila. Hydrobiologia, 215 : 13-19.

Tedesco M.A. \& Duerr E.O. 1989. - Light, temperature and nitrogen starvation effects on the total lipid and fatty acid content and composition of Spirulina platensis UTAX 1928. J. applied Phycol., $1:$ 201-209.

Thompson P.A., Guo M-x. \& Harrison P.J. 1992. - Effects of variation in temperature. I. On the biochemical composition of eight species of marine phytoplankton. J. Phycol., $28: 481-488$.

Vargas M.A., Moreno R.J., Olivares H., Del Campo J.A., Rivas J. \& Guerrero M.G. 1998. - Biochemical composition and fatty acid content of filamentous nitrogen-fixing cyanobacteria. $J$. Phycol., $34: 812-817$.

Wallen D.G. \& Geen G.H. 1971. — Light quality and concentration of proteins, RNA, DNA and photosynthetic pigments in two species of marine plankton algae. Mar. Biol., $10: 44-51$.

Zhu C.J., Lee Y.K. \& Chao T.M. 1997. - Effects of temperature and growth phase on lipid and biochemical composition of Isochrysis galbana TK1. J. applied Phycol., 9 : 451-457. 